\title{
EL DESARROLLO ECONÓMICO Y LOS RECURSOS HUMANOS EN MÉXICO: UN ESQUEMA CONCEPTUAL
}

\author{
JosÉ B. MoRELOS \\ El Colegio de México
}

\section{INTRODUCCIÓN}

Los elementos estructurales que configuran el esquema polar desarrollo-subdesarrollo son esencia y consecuencia de la interacción y funcionamiento de las diversas estructuras de los sistemas. La interdependencia y complementariedad de las diversas estructuras están relacionadas con los patrones de acumulación de capital, la forma de participación de los agentes económicos, los patrones de apropiación del producto y los procesos de estratificación y movilidad social -factores que se encuentran interrelacionados con el comportamiento del mercado de trabajo y sus imperfecciones. Por ello el conocimiento de los síntomas y de los elementos sustantivos de una de las variables polares: el subdesarrollo, es condición necesaria para el estudio de los recursos humanos que, por su naturaleza y función, constituyen una parte esencial del proceso de desarrollo de los países; e igualmente importante para la formulación de estrategias y políticas en materia de absorción de empleo y formación de los recursos humanos.

El propásito del presente trabajo es mostrar algunos aspectos del desarrollo económico de México que sirvan de marco de referencia para establecer una tipología $y$, con base en la misma, discutir los factores que conforman el esquema conceptual del estudio de los recursos humanos.

\section{Características del desarrollo económico de MéXico}

El carácter hegemónico de las economías centrales no ha perdido su significación en el proceso de desarrollo de las economías latinoamericanas. La relación de dependencia existente en el modelo primario exportador tiene vigencia, aunque en forma más compleja, en el modelo de industrialización por sustitución de importaciones. ${ }^{1}$

Durante el período de crecimiento "hacia afuera" de las economías latinoamericanas se cristalizó su surgimiento, expansión e integración

1 Osvaldo Sunkel, "Subdesarrollo, Dependencia y Marginación: Proposiciones para un Enfoque Integrado". Documento presentado en el Seminario de La Marginalidad en América Latina, organizado por el Banco Interamericano de Desarrollo (BID) y la Sociedad Chilena de Planificación y Desarrollo (PLANDES), Santiago, Chile. 
al sistema económico internacional. Los cambios estructurales que experimentan las economías centrales transforman la estructura de algunos sectores de las economías periféricas; asimismo se verifica una reorientación de las políticas gubernamentales, las que tienden a beneficiar al sector exportador.

A finales del siglo XIX, en México, las medidas de política económica del Porfiriato - aranceles, importaciones libres de derechos, exenciones de impuestos fiscales- estaban orientadas al fomento del desarrollo industrial. De igual manera se favoreció a la minería. Entre 1892 y 1907 la tasa de crecimiento de la industria manufacturera fue de $9.4 \%$ y un poco menor la de la industria minero-metalúrgica. La agricultura durante esta época se caracteriza por su escaso dinamismo, destacando también la ausencia de una clase empresarial cuyos efectos fueron la incapacidad del sector agropecuario para absorber cambios tecnológicos y elevar su productividad. ${ }^{2}$

La reorganización de la producción y el progreso tecnológico se verifica años más tarde al llevarse a cabo la reforma agraria. Lo anterior fue posible gracias a la acción que en esta dirección llevó a cabo el nuevo grupo político, acción que se identificó con la expansión de la infraestructura económica y social, el apoyo a la pequeña propiedad y el establecimiento de políticas de riego -elementos importantes de las políticas de fomento agropecuario. ${ }^{3}$

Todas estas medidas provocaron un crecimiento rápido del sector agropecuario, que coincidió con el período de desarrollo con inflación. La tasa de crecimiento del sector en el período 1946-1956 fue de $7.6 \%$.

El desarrollo económico de México se vio favorecido por el desarrollo agrícola. Su contribución al desarrollo del país está dada en términos de su capacidad para producir bienes para el consumo interno -finales e intermedios - y para el mercado externo, proveyendo así divisas para cubrir las importaciones de bienes de producción, aumentar la capitalización del país y ampliar la base productiva del mismo.

Hacia el decenio de los 50 termina la fase de crecimiento "hacia afuera" y a partir de este año el sector agropecuario se caracteriza por su virtual estancamiento. En el período 1957-1967 la tasa de crecimiento descendió a $3.8 \%$.

La desaceleración del ritmo de crecimiento del sector agropecuario fue consecuencia, entre otros factores, del cambio de orientación de la inversión pública que motivó la asignación de menos recursos a las obras de fomento agropecuario; de la relación de precios internos, que fue desfavorable al sector, y de la depresión de los mercados internacionales de productos primarios iniciada con la crisis de 1929.

A partir de los años 50, México entra abiertamente en la etapa de industrialización por sustitución de importaciones (crecimiento hacia adentro). Conviene mencionar, sin embargo, que el desarrollo de las manufacturas marchó al paso con el del sector agropecuario. Desde 1935 las manufacturas logran aumentos de importancia en sus tasas

2 Leopoldo Solis, "Hacia un análisis general a largo plazo del desarrollo económico de México", DEMOGRaff́ y eCONOMía, Vol. I, Núm. 1. 1967, pp. 40-52.

3 Solís, loc. cit. 
de crecimiento, sobrepasando el $8 \%$ en los años que siguen a 1946. En el período 1940-1962 las manufacturas aumentan su participación en el producto bruto interno al pasar de $17.8 \%$ al $23.3 \%$.

El desarrollo del sector manufacturero, como los de electricidad, petróleo y construcción, se vieron favorecidos por el sector gubernamental al proteger y alentar su modernización con diversas medidas. Destacan por su importancia las políticas de fomento económico, exenciones de impuestos, protección arancelaria, facilidades para ampliar la base productiva del país; el establecimiento de mecanismos financieros - políticas de control selectivo del banco central y políticas financieras de las instituciones nacionales-; la transformación favorable al sector de la matriz de precios internos; subsidios al consumo de la población urbana; el comportamiento del sector externo $\mathrm{y}$ en general las políticas de sustitución de importaciones.

EI verse la agricultura parcialmente desfavorecida al mismo tiempo que el sector industrial centralizaba la atención de la política económica, fueron condiciones que junto con la reforma agraria aceleraron la transferencia de la mano de obra hacia los sectores urbanos.

La expansión de las actividades industriales por las que atravesaba el país permitió absorber la mano de obra proveniente de las áreas rurales o de las localidades intermedias.

La absorción del excedente de mano de obra por la industria en una primera etapa probablemente no creó problemas de empleo, aunque sí afectó los salarios reales en el mercado de trabajo urbano. ${ }^{4}$ Posteriormente, al ser incapaz el desarrollo industrial — por falta de expansión, por la tecnología o por las características de la ofertade absorber la mano de obra que fluía del campo a la ciudad, se creó un nuevo tipo de excedente. En opinión de Pinto, este excedente constituye el marginalismo urbano que surge de la incapacidad de las actividades básicas para absorber mano de obra, del crecimiento demográfico y del desajuste entre el crecimiento urbano y el rural. ${ }^{5}$

Tanto el excedente rural originado por la indivisibilidad de la tierra y por el tamaño promedio de la familia rural, como el urbano, tienen repercusiones distintas en la economía. Desde el punto de vista del costo social el excedente rural es menos oneroso que el urbano. El primero se sustenta en una forma embrionaria del bienestar social basado en la familia, mientras que el segundo normalmente desocupado o subocupado tiene un costo social más elevado, pero su bajo nivel de ingreso es compensado por las facilidades sociales que le brinda la ciudad.

Ambos excedentes son algunas de las manifestaciones directas o indirectas de la vinculación de las economías trasnacionales, de la industrialización orientada fundamentalmente hacia la producción de bienes finales de consumo, y de la concentración industrial en los grandes centros urbanos.

La desnacionalización y la sucursalización de la industria mexicana,

4 David Ibarra, "Mercados, desarrollo y política económica", en El Perfil de México en 1980, I, México, Siglo XXI, 1970, p. 118.

5 Aníbal Pinto, "En torno a Chile -una economía difícil", El Trimestre Económico, Vol. XXXIII (2), Núm. 130, abril-junio, 1966, p. 174. 
que se han acelerado en el último decenio, son los rasgos distintivos de la nueva forma de dependencia. El cambio en las modalidades, como menciona Sunkel, "de captar y absorber la contribución externa de recursos productivos es uno de los elementos más importantes que han incidido... en los resultados que exhibe el proceso de industrialización en cuanto a su influencia sobre el ritmo de crecimiento de la economía, el nivel de ocupación y la marginación, la distribución del ingreso, la selección de líneas de producción...". ${ }^{6}$ Si bien estas consideraciones se hacen con referencia a América Latina, se piensa que también son aplicables al caso mexicano.

Aspectos del desarrollo regional. En los últimos cuarenta años, los sectores más dinámicos del desarrollo económico de México han sido la agricultura y la industria. La primera lo fue durante 15 años o sea hasta mediados del decenio de 1950; y la industria a partir de la segunda Guerra Mundial. La ubicación de Ios sectores más dinámicos en ciertas áreas geográficas del país es lo que ha determinado su desarrollo económico.

El trato preferencial que en algunos aspectos han recibido los sectores estratégicos, y la mayor importancia dentro de los mismos de los sectores modernos, es lo que explica el mayor desarrollo de ciertas regiones, que en opinión de Mendoza son regiones emergentes; ${ }^{7}$ pero de acuerdo con el contexto del presente trabajo se denominan regiones hegemónicas o dominantes.

El desarrollo regional, por lo tanto, no se basa sólo en el predominio de actividades estratégicas, sino dentro de éstas en la mayor o menor importancia de las actividades moderna o tradicionales. De igual modo, las políticas gubernamentales de carácter selectivo, en términos regionales, $\mathrm{y}$ de fomento agropecuario e industrial, han contribuido al desarrolio rápido y sostenido de las actividades modernas agrícolas e industriales. La mayor importancia de éstas en determinadas regiones o entidades ha provocado las diferenciales en el nivel de ingreso regional, en el proceso de urbanización, en la composición sectorial y ocupacional de la fuerza de trabajo y en la disponibilidad de servicios sociales y asistenciales.

En 1950, en el 56.3\% de las entidades predominaba la agricultura de subsistencia y en 1960 este porciento disminuyó a $50 \%$. Las entidades con agricultura moderna significaron el 18.8 y el $31.3 \%$ en cada uno de estos años. ${ }^{\mathrm{s}}$

Para caracterizar la agricultura moderna, entre otros elementos, se utilizan el tipo de relaciones: capital/trabajo y producción/tierra. ${ }^{9}$ En 1960 la relación capital/trabajo del sector tradicional representaba el $38.3 \%$ respecto a la del moderno y $26.9 \%$ para 1960 . La relación

6 Sunkel, op. cit.

7 Eliseo Mendoza Berrueto, "Implicaciones regionales del desarrollo económico de México", DEMografta Y ECONOMía, Vol. III, Núm. 1, 1969, pp. 25-63.

8 Se distinguen tres tipos de agricultura: moderna, tradicional y en transición. Véase Saúl Trejo et al., "Dualidad económica de la agricultura mexicana". Departamento de Estudios Económicos, Banco de México, 1970. (Circulación interna.)

9 Los otros son: técnicas mecanizadas, fertilizantes y riego y superficie. Trejo et al., op. cit. 
producción/tierra fue en 1950 de $44.1 \%$ respecto al moderno y aumentó a $58.9 \%$ para 1960.

Entre 1950 y 1960, la fuerza de trabajo en el sector tradicional aumentó en un $71.1 \%$ y el aumento en el sector moderno fue de $138.1 \%$. En los años analizados el sectur agrícola moderno ha tenido un crecimiento más dinámico en cada uno de los factores de producción, siendo sólo aventajado por el tradicional en la relación producción/tierra. Las elasticidades capital/producto y producción/mano de obra $^{10}$ fueron para el sector urbano de 1.5 y 1.9 , y de 1.3 y 1.6 para el tradicional.

Las entidades o regiones en que predomina la agricultura tradicional son en general las menos desarrolladas y cuyos niveles de ingreso per capita y de bienestar están muy por debajo de aquellas entidades con agricultura moderna. Estas últimas entidades tienen más altos porcientos de población en localidades urbanas y sus ciudades son las que registran el más alto crecimiento demográfico. Asimismo estas regiones son las más beneficiadas con la política de riego.

El sector manufacturero al igual que el agrícola presenta la característica de dualidad. Existen indicios, con base en el comportamiento observado, de que la tendencia del sector tradicional es la de participar menos en el producto total. El sector moderno absorbía en 1960 el $42.3 \%$ del total de la mano de obra, porciento que aumentó a 50.9 en 1965. Paralelamente al aumento del empleo la productividad se elevó, lo que puede ser debido a los cambios tecnológicos. ${ }^{11} \mathrm{El}$ sector tradicional, por el contrario, registra disminuciones tanto en el porciento de empleo como en la productividad: en 1960 el valor agregado por trabajador fue de 4231 pesos y en 1965 de 3411 . Asimismo disminuyó, como ya se mencionó, su participación en la producción total manufacturera. En 1960, su participación fue de $12.6 \%$ y en 1965 de $7.7 \%$.

Las ramas industriales de corte moderno se encuentran ubicadas por lo general en los centros urbanos densamente poblados, lo que ha contribuido a fortalecerlos y provocar a su vez un trato preferencial por el gobierno y los grupos empresariales en términos de inversiones en infraestructura social y en inversiones productivas, con lo que se ha fomentado, deliberadamente o no, la concentración industrial y urbana en determinadas entidades o ciudades.

En el sector servicios coexisten también actividades altamente productivas con aquellas, bastante numerosas, de productividad muy baja.

La importancia del subsector tradicional dentro de las actividades terciarias presenta, a nivel de entidad, un rango de variación menos amplio que el observado en el industrial. En 1960, a las actividades tradicionales correspondía el $57.6 \%$ del empleo total en servicios y en 1965 el $51.9 \%$.

Las actividades tradicionales se encuentran concentradas en el comercio y otros servicios, pero esta última rama genera más del $60 \%$ del valor agregado del sector.

10 La elasticidad se obtuvo al relacionar el incremento del capital respecto al incremento de producción, y el incremento de producción respecto al incremento de mano de obra.

11 Saúl Trejo, et al., op. cit. 
La baja participación del comercio de tipo tradicional (19.0\% y $21.7 \%$ en 1960-1965) unido a que en este sector existe una alta proporción de la ocupación, indica que el nivel de ingreso per capita es muy reducido y asimismo corresponde a este sector una elasticidad (valor agregado/producción) menor de 1, por lo que los aumentos en la producción bruta no se han traducido en aumentos proporcionales en el valor agregado.

La falta de información para fechas más remotas no permite determinar cuál ha sido la dinámica de los sectores tradicionales y modernos, su comportamiento en los períodos de crecimiento hacia adentro y hacia afuera y la importancia que uno $u$ otro han tenido en el desarrollo regional del país. Lo que parece claro es que las diferencias regionales obedecen, además del dinamismo del sector o sectores estratégicos, a la importancia relativa que dentro de éstos han tenido los sectores modernos, y que el desarrollo de éstos ha sido, entre otros factores, lo que explica en parte el crecimiento de ciudades que se sustentan en la agricultura comercial y en la manufactura de corte moderno. Como corolario de lo anterior, aquellas entidades en que predomina la agricultura y manufactura tradicionales son las que han quedado a la zaga tanto en su desarrollo como en su proceso de urbanización. Conjuntamente con lo anterior, el trato preferencial del gobierno y de los grupos empresariales en términos de atención, recursos y localización de empresas en determinadas entidades ha creado las condiciones para la existencia de entidades dominantes y dependientes.

\section{TIPOLOGÍA URBANO-RURAL}

La ausencia de un desarrollo regional armónico se tipifica por el comportamiento del índice de concentración. ${ }^{12}$ En 1940 el índice de concentración fue de 18.3 y en 1960 de 21.3.

La concentración ha dado origen a la disminución del número de entidades de desarrollo económico rápido. En 1960 existían 14 entidades y 8 en 1960, representando en términos relativos el 43.7 y el $25.0 \%$, respectivamente. ${ }^{13}$

Lo anterior es un indicador de la forma en que se ha polarizado el desarrollo económico regional. Por un lado, pocas regiones o entidades dominantes, y por otro regiones o entidades dependientes.

El uso de variables polares implica la existencia de un continuum en los niveles y grados de dominación o dependencia.

Las áreas dominantes son el resultado de condicionamientos históricos internos y externos, de las políticas gubernamentales y de la orientación y monto del gasto público y de la acción de los grupos empresariales nacionales y extranjeros, factores que han permitido ampliar la base económica de dichas áreas y provocado la diversificación de la economía.

Operacionalmente es posible identificar la dominación con la diver-

12 Luis Unikel y Edmundo Victoria, "Medición de algunos aspectos del desarrollo socioeconómico de las entidades federativas de México, 1940-1960", DEMoGRAFÍA Y ECONOMíA, Vol. IV, Núm. 1, 1970, pp. 1-42.

13 Ibid. 
sificación de la economía y con el predominio de sectores modernos. En este sentido se puede hablar de relación directa entre los factores antes mencionados. De esta forma, las áreas dependientes serían aquellas que se caracterizan por ser predominantemente unifuncionales siendo su actividad básicamente tradicional.

Las actividades predominantes que expresan la rama o ramas de actividad en que se especializan las economías regionales son un indicador del grado de desarrollo económico. Normalmente, las áreas de economía diversificada se identifican con las de más alto nivel de desarrollo económico y de ritmo y grado de urbanización.

Trasponiendo estas ideas al plano urbano-rural, su carácter deviene de la presencia o no de sectores dinámicos o estratégicos, de la diversificación de su economía y, dentro de ésta, del predominio de las actividades modernas $y / 0$ tradicionales $y$ del trato preferencial dado por el gobierno, los grupos empresariales y las instituciones financieras nacionales e internacionales.

La interacción de estos factores facilita la presentación de un modelo de tipo ideal, utilizando variables polares: dominación y dependencia, las que están interrelacionadas con la acción de los agentes económicos.

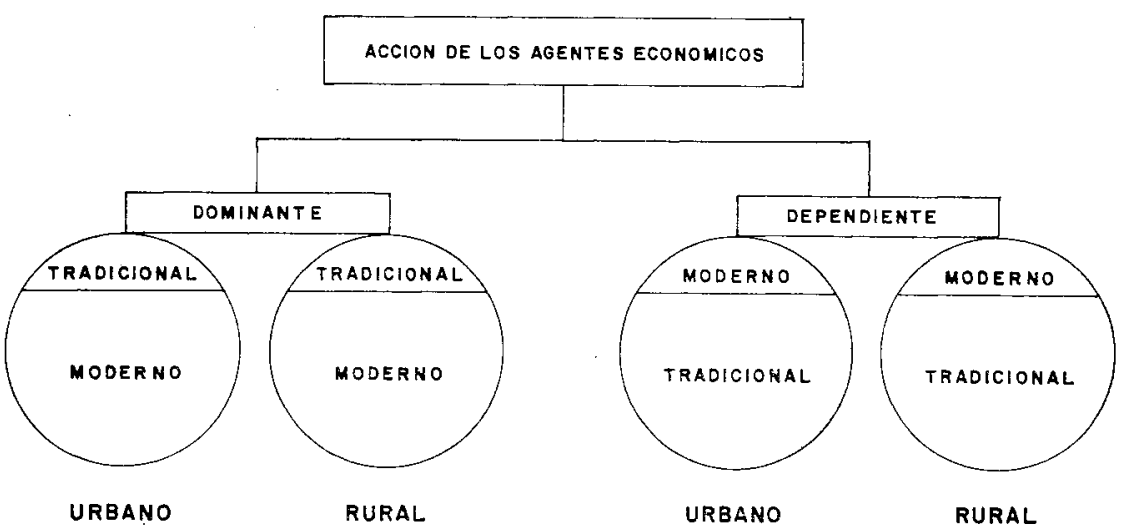

La existencia urbana tiene su base en el juego de los agentes económicos a lo largo del desarrollo histórico de la sociedad. El trato preferencial que otorgan dichos agentes se manifiesta en la proporción relativa de las inversiones sociales y productivas encaminadas a ensanchar la base económica de las localidades urbanas dominantes. Lo mismo puede decirse del tipo de política como: precios relativos favorables, subsidios al consumo de la fuerza de trabajo y precios inferiores en algunos insumos industriales (energía eléctrica), exenciones de impuestos, etc. Por parte del sector empresarial el trato desigual está dado por el establecimiento de industrias y comercios en estas áreas por las ventajas que ello les representa: economías externas y aprovechamiento de la infraestructura. 
Consideraciones semejantes pueden formularse con el sector dominante rural que presenta como aspecto fundamental la existencia de un sector agrícola básicamente moderno. La atención en términos de fomento agropecuario resulta mayor que la de aquellos sectores rurales donde la actividad predominante es la agricultura tradicional.

Tanto en los sectores urbano y rural dominantes, como se ve en el diagrama, coexisten con las actividades modernas las tradicionales, pero el peso de estas últimas es menor en términos del valor de la producción bruta y del valor agregado por trabajador.

Los sectores dependientes se consideran complementarios, que hasta cierto punto dan forma a los sectores dominantes. Su existencia, interdependencia y complementariedad está dada también por condicionamientos históricos.

Las localidades urbanas dependientes son aquellas en que las actividades predominantes son las tradicionales. Asimismo la falta de una dinámica hacia la modernización, consecuencia de la acción de los grupos internos o externos a la región y su enclave geográfico, son factores que explican dicho carácter. Su condición de dependiente puede venir del trato descriminatorio de los agentes económicos o bien por factores externos. Por ejemplo, áreas urbanas que son cen. tros comerciales que se sustentan en la agricultura tradicional, o bien lacalidades en las que predominaba la minería y que al decaer esta actividad se tradujo en el estancamiento de los centros urbanos.

La existencia de sectores dominantes y dependientes ${ }^{14}$ viene a decir las diferencias en niveles de ingreso inter e intra sectores; patrones y composición del consumo y de la inversión; contenido de educación y calificación de la mano de obra; patrones de migración; tipo de oportunidades para lograr una movilidad ascendente; distinta composición sectorial y ocupacional, patrones igualmente diferenciales en fecundidad y mortalidad y crecimiento demográfico. Asimismo los sectores dominantes, además de tener mayor acceso a las fuentes de financiamiento, beneficios sociales y toma de decisiones, se encuentran asociados con los centros trasnacionales existiendo, por lo tanto, una identificación en patrones de consumo, ingreso, etc. con los de los grupos de las economías centrales. ${ }^{15}$

\section{ESOUEMA PARA EL ESTUDIO DE LOS RECURSOS HUMANOS}

En las consideraciones que se han formulado anteriormente se ha subrayado el papel de los factores económicos. Esto no quiere decir que se esté olvidando el papel de los otros factores, ya que la concepción del desarrollo debe comprender aspectos políticos, sociales, culturales y económicos.

El esquema que se presenta se basa en la interdependencia que existe entre las distintas estructuras con el desarrollo conómico y social y los recursos humanos.

14 Algunos autores utilizan en vez de la noción de dependiente la de marginación o marginal. Véase Sunkel, op. cit. y Roger Veckemans, "Marginalidad y Pleno Empleo", trabajo presentado en el Seminario sobre La Marginalidad en América Latina, organizado por BID-PLANDES, Nov. 1970, Santiago, Chile.

15 Veckemans, op. cit.; Sunkel, op. cit. 
Los tipos de estructuras que se han incluido son: la cultural, la social, la económica y la de poder, las cuales están estrechamente vinculadas con el comportamiento de la oferta y la demanda de recursos humanos. Dentro de cada estructura se han incluido aquellos factores que se consideran más relevantes y que tienen una estrecha relación con la composición tanto cuantitativa como cualitativa del factor humano (véase el diagrama siguiente).

En este apartado además de presentar una descripción del esquema se buscará introducir algunas notas sobre el comportamiento de los diversos factores en cada uno de los tipos propuestos antes.

Por consideraciones metodológicas el esquema puede dividirse en tres niveles: 1) nivel global; 2 ) nivel intermedio, y 3 ) nivel individual.

Nivel global. A este nivel se estudia la interdependencia de los cuatro tipos de estructuras con la oferta y la demanda de los recursos humanos. ${ }^{16}$ El esquema no pretende ser exhaustivo, por lo que en cada una de las diversas estructuras se mencionan aquellas variables que se consideran vinculadas con el comportamiento de los recursos humanos.

Dentro de la estructura social se han incluido: la estratificación social, la estructura de clase y la movilidad social. En la económica: el capital, el ahorro, la producción y la tecnología. Con la estructura de poder están asociadas las políticas gubernamentales como: las políticas de industrialización, físcal, arancelarias, infraestructura y de legislación laboral.

El comportamiento de las distintas estructuras va a tener a su vez una repercusión directa sobre el crecimiento demográfico y sobre la composición ocupacional y educacional de la población.

Las variables demográficas van a determinar tanto el tamaño como el crecimiento y distribución geográfica de la mano de obra, asimismo el volumen y composición por edad y sexo de las entradas y salidas. El flujo de las entradas y salidas dependerá, además, de los factores económicos y de la capacidad del sistema educativo para absorber y retener a la población dentro del sistema. Del mismo modo la influencia de la estructura social a través de la estratificación social, la estructura de clase y la movilidad social serán también pertinentes en el flujo de las entradas y salidas y en la composición de la mano de obra por sectores o ramas de actividad y por ocupaciones. Igualmente estos factores están ligados con el acceso de la población a los distintos ciclos educativos y a la permanencia de la misma.

En el aspecto educativo se analizarían las políticas gubernamentales en materia de educación, el monto y la compósición de la inversión en educación, elementos que vienen a decir del papel que juega la educación institucional en la transmisión de conocimientos, creación de actitudes, valores y motivaciones encaminadas a impulsar el desarrollo y en la formación directamente de los recursos humanos.

Entre las distintas estructuras existen relaciones de causa-efecto o simplemente funcionales. Por ahora es difícil determinar cuales

16 En el esquema no se ha introducido la variable exógena: influencia de las economías centrales sobre el desarrollo económico del país. Algunas consideraciones sobre este punto se presentarán en el texto. 
son las relaciones causales, especialmente por la complejidad de las interrelaciones.

Nivel intermedio. El estudio de los recursos humanos a este nivel se ha dicotomizado. Por un lado, se estudia la unidad de demanda (empresa) y, por otro, la unidad de oferta (la familia).

La unidad de demanda se estudia como una institución que realiza actividades en relación con la producción de bienes y servicios. Esta institución en cuanto a sus características está conformada por las distintas estructuras.

El estudio de la empresa como agente productor comprende los siguientes aspectos: la estructura económica de la empresa $y$, asociada a ésta, sus niveles de productividad y su estructura tecnológica ; ambos aspectos determinarán la forma en que se combinan los insumos productivos: capital y trabajo. Asimismo, por la importancia que tienen en el funcionamiento de la empresa, en su estructura y en sus niveles de eficiencia se estudian las condiciones de trabajo: características físicas, relaciones humanas, etc. En cuanto a criterios de reclutamiento, dentro de este punto, se analizan las políticas de la empresa en cuanto al personal, pero esto a su vez dependerá de que exista la organización sindical y del tipo de ésta, de la utilización de agencias de empleo para reclutar personal, etc.

De las condiciones de trabajo y de las políticas de reclutamiento van a depender los niveles de ausentismo, y rotación, los que se incluirán en la eficiencia y funcionamiento de la empresa. En relación con estos aspectos se estudiará el costo económico y las repercusiones de éste en la empresa. Asimismo, los problemas de pirateo y la importancia de las prestaciones sociales y de tipo de prestaciones sociales, asi como la finalidad de las mismas.

La unidad de oferta es la familia, ya que es el núcleo básico proveedor de recursos productivos y el agente primario de socialización.

El análisis de la familia se hará desde el punto de vista cuantitativo y cualitativo. Las características cuantitativas que se estudiarán son: tamaño y composición por sexo y edad de la familia; número de miembros que participan en la producción de bienes y servicios; nivel socioeconómico, en el que se analizan el nivel de ingreso familiar, el nivel educacional de sus miembros y el nivel ocupacional de los mismos; condiciones de vivienda y salud. Las características cualitativas: la estructura de valores de la familia.

Tanto las características cuantitativas como las cualitativas van a determinar las oportunidades educacionales y ocupacionales de cada uno de sus integrantes, el flujo de entradas y salidas a la actividad y la movilidad social. Esta se estudiará bajo los siguientes enfoques: movilidad vertical, movilidad horizontal y movilidad intergeneracional. Las características familiares van a influir en el tamaño y composición de la oferta de mano de obra, y asimismo las características ocupacionales y educativas de los miembros que participan en la producción de bienes y servicios en la composición ocupacional de la fuerza de trabajo.

Nivel individual. En este último punto se estudiarán los aspectos objetivos y subjetivos de las personas. Las características objetivas 


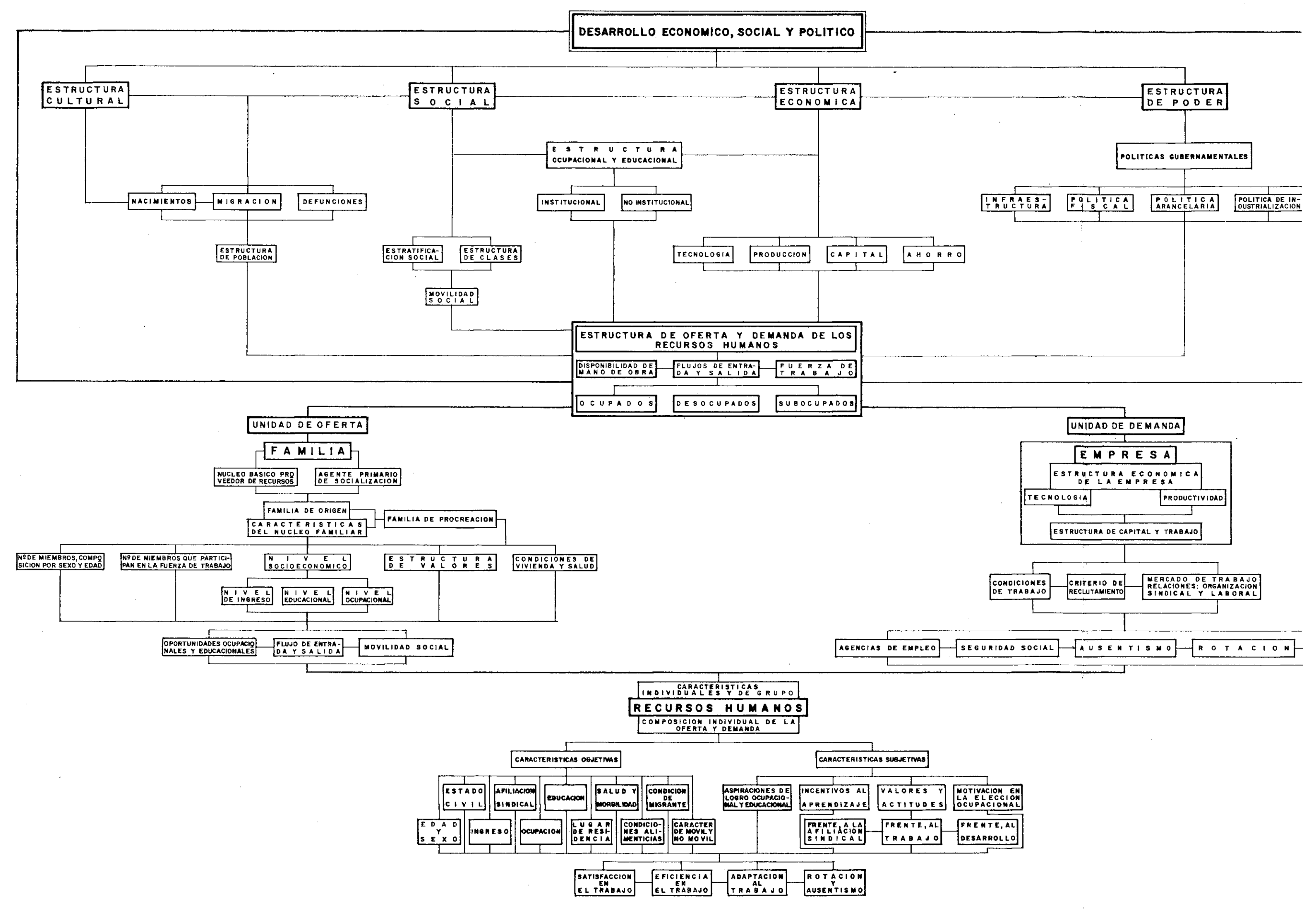


son: edad y sexo, estado civil, ingreso, afiliación sindical, ocupación, educación, lugar de residencia, salud, alimentación, condición de migrante y carácter de móvil o no móvil.

Las características subjetivas son: aspiraciones de logro ocupacional, incentivos al aprendizaje, valores y actitudes, comprendiendo en este punto la actitud frente a la afiliación sindical, al trabajo y al desarrollo.

Tanto las características cuantitativas como las cualitativas están correlacionadas con la satisfacción en el trabajo, con su eficiencia en el mismo, con la adaptación y finalmente con la rotación y el ausentismo (véase el diagrama).

\section{CONSIDERACIONES EN TORNO A LA TIPOLOGÍA Y ALGUNAS VARIABLES DEL ESOUEMA}

Las notas que siguen tienen como objetivo analizar sólo puntos particulares del esquema teniendo como punto de referencia el modelo de tipos ideales anteriormente propuesto.

El carácter de sectores dominantes de las áreas urbanas y rurales los sitúa en condiciones ventajosas frente a las dependientes, ventajas que se derivan de la concentración de la industria fabril, de los servicios educativos, de su capacidad para influir en las decisiones de política económica, de su acceso a las fuentes financieras nacionales e internacionales, etc.

La centralización de la industria y el predominio de actividades de corte moderno, además de ampliar la base productiva de estas economías, están asociadas con la capacidad endógena de crecimiento, la que se sustenta en la generación de excedentes que les permiten disponer de adelantos tecnológicos y organizacionales y asimismo determinar el ritmo y orientación del crecimiento económico.

La situación se torna más desfavorable si lo anterior se asocia al proceso de desnacionalización y sucursalización de la industria mexicana. ${ }^{17} \mathrm{La}$ industria trasnacional no sólo impone pautas al ritmo y orientación del crecimiento industrial dentro del sector sino que también ocasiona desequilibrio en el mercado de trabajo al provocar un aumento de la demanda de personal calificado y disminuir la del personal sin calificación lo que tiende a deprimir los salarios reales de estos últimos. Lo anterior también es consecuencia de las tasas de crecimiento de la población y de la urbanización. ${ }^{18}$

El imponer pautas en el crecimiento dentro del sector de la industria trasnacional ha contribuido a la diversificación y modernización de los procesos industriales de la empresa mexicana que contaba con la capacidad para introducir dichos cambios. Pero la penetración de la industria con intereses trasnacionales ha afectado a la industria artesanal que no puede hacer frente a la competencia o que por no servir

$17 \mathrm{La}$ inversión extranjera directa a partir de 1950 ha estado creciendo en la industria manufacturera a una tasa de $14.0 \%$. Para mayor detalle véase: Víctor L. Urquidi y Adrián Lajous Vargas, Educación superior, ciencia y tecnología en el desarrollo económico de México: un estudio preliminar, EI Colegio de México, México, 1967, p. 17.

18 Ibid., p. 19. 
de apoyo logístico a la industria moderna desaparece del mercado. Es muy posible que parte de la mano que por tal motivo queda desplazada no sea absorbida por la industria moderna, factor que también contribuye a deprimir los salarios reales.

La diversificación y modernización de la economía de estas regiones ha modificado la composición funcional por sectores y ocupaciones de la fuerza de trabajo, hecho que se manifiesta en la concentración de los profesionistas, técnicos, y obreros calificados y en la proporción de la población ocupada en los sectores no agrícolas. A este respecto, "más de un tercio de los profesionistas y técnicos se encuentran en el Distrito Federal y en esta entidad reside del 40 al $50 \%$ de los ocupados en manufacturas, construcción, electricidad, gas y comercio". 19

Respecto a los servicios educativos, éstos se encuentran concentrados básicamente en las principales áreas urbanas de corte dominante que existen en el país. Por ello no resulta aventurado decir que el acceso a la educación superior es privativo de algunos de los estratos sociales que pertenecen a los sectores modernos: dominantes y dependientes.

En términos de eficiencia educativa, ésta es mayor en las áreas urbanas y muy posiblemente esté también concentrada en las áreas urbanas dominantes. Lo mismo se puede decir en cuanto al monto y orientación de la inversión en educación, básicamente por el crecimiento demográfico de estas localidades y por la demanda efectiva de educación.20 Por lo que corresponde a la enseñanza no institucional, el tamaño de las empresas, su capacidad económica y la necesidad de adaptar su mano de obra a los cambios en sus procesos productivos, favorecen también a la población trabajadora de las áreas urbanas dominantes.

El contenido de educación de la población, sus mayores posibilidades de acceso y permanencia, sus niveles de ingreso y el predominio de cierto tipo de ocupaciones son elementos que inciden en los procesos y patrones de estratificación, estructura de clase y movilidad social que imperan en las áreas dominantes, factores que a su vez están relacionados con los patrones de reproducción, mortalidad y morbilidad. En estas regiones las normas y valores que tienen los distintos grupos sociales frente al reemplazo físico de sus miembros son más pluralistas que las que existen en las localidades de tipo dependiente. De esta forma el comportamiento demográfico de ambas poblaciones es distinto, y lo será por lo tanto el volumen y composición de la población que se incorpora a la actividad. En las áreas dominantes la población se incorpora a edades más avanzadas y su contenido en términos educativos es mayor que el de las áreas dependientes.

El mayor poder de los grupos sacioeconómicos de los sectores dominantes se manifiesta en el acceso que tienen a los grupos de poder en la toma de decisiones y en la fijación de las políticas gubernamentales. Derivado de lo anterior, las áreas dominantes se ven

19 Ibid., p. 29.

20 Ibid. 
mayormente favorecidas en términos de atención y trato preferencial por lo que respecta a inversiones en infraestructura, políticas arancelarias y fiscales y acceso a las fuentes de financiamiento nacionales e internacionales.

En las áreas dependientes, por el contrario, su crecimiento económico tiene el carácter de inducido. La mayor importancia relativa de las actividades tradicionales hace que la combinación de factores sea distinta a la observada en las áreas dominantes, básicamente las de corte moderno. En éstas la industria es intensiva en capital, con altos niveles tecnológicos y mayores niveles de sueldos y salarios. Las zonas rurales dependientes se sustentan en la agricultura tradicional, sus niveles de subempleo son elevados y sus niveles de ingresos muy bajos.

En términos de los servicios educativos, las regiones dependientes están en posición de desventaja no obstante los esfuerzos gubernamentales para ampliar la educación y disminuir los niveles de analfabetismo. Esto supone que se han dado las facilidades necesarias en términos de servicios para que la población tenga acceso al sistema educativo, pero su acceso y permanencia finalmente están determinados por los niveles de ingreso de la población por cuyo motivo en muchos de los casos sale del sistema educativo para incorporarse a la fuerza de trabajo.

En lo demográfico, las regiones dependientes difieren de las dominantes - sus niveles de crecimiento natural son más elevados, pero su crecimiento total es inferior por efectos de la migración. La población migrante no tiene normalmente las calificaciones o el contenido educacional que se demanda en las regiones de destino (dominantes), dadas las condiciones estructurales de la modernización que incide en la absorción de mano de obra calificada, quedando la sin calificación frecuentemente desempleada o subempleada. Esta mano de obra desempeña normalmente ocupaciones inestables y de bajo nivel de productividad.

La falta de integración de estos sectores a los centros institucionales o con los grupos dominantes o gobierno tiene implicaciones en las dificultades para obtener créditos y en la menor atención de los gobiernos en términos de inversiones en infraestructura y de política económica.

Lo hasta ahora mencionado permite extraer algunos comentarios finales.

En primer lugar, al realizar el análisis a nivel regional queda de manifiesto el carácter heterogéneo de la economía por lo que se refiere a las actividades predominantes, composición ocupacional, servicios educativos, niveles de salarios, combinación de los factores de la producción y niveles tecnológicos. Este último factor reviste particular importancia cuando se realizan proyecciones de demanda de mano de obra por el método tradicional. No es suficiente definir la cantidad de capital necesaria para emplear, a nivel medio de productividad, a un trabajador y posteriormente pasar a estimar la tasa de inversión necesaria para dar ocupación a una población activa creciente. Un enfoque más realista, suponiendo heterogeneidad de los niveles de tecnología, sería el de prever el desarrollo tecnológico de 
los sectores modernos nacionales y trasnacionales y el de los tradicionales. Si el primero avanza más rápido que el segundo es probable que la supresión de empleos sea mayor que la creación, lo que generaría desempleo y subempleo. Asimismo serían mayores las demandas de recursos humanos con ciertos niveles de calificación $\mathrm{y} / \mathrm{o}$ contenido educativo. Esto a su vez incidiría en los salarios reales y en la concentración del ingreso, con los efectos concomitantes que de tal situación se deriva.

La diferencia en los adelantos tecnológicos se ajusta a la realidad nacional, lo que queda de manifiesto en el modelo de tipos ideales.

Para que la modernización genere desempleo o subempleo es necesario que se cumpla, entre otras, la siguiente condición: que la población ocupada en el sector tradicional sea relativamente elevada. Esta condición tiene validez en el caso de México. Las actividades tradicionales tanto a nivel geográfico como sectorial representan alrededor del $50 \%$.

En segundo lugar, la planeación de la educación y la formulación de políticas tienen que estar referidas a las modalidades que presente el desarrollo económico del país. Es decir, la planeación y las políticas deben basarse en los elementos sustantivos que definen a nivel regional las características estructurales del binomio desarrollo-subdesarrollo que caracteriza a la economía mexicana. Y, por último, el sistema educativo, además de formar directamente a los recursos humanos, debe ser un medio que permita a la población adaptarse a las instituciones cambiantes de la sociedad. 